\title{
NONLINEAR EFFECTS IN WAVE SCATTERING AND GENERATION
}

\author{
Flow Interaction with Topography
}

\author{
Roger Grimshaw \\ Department of Mathematical Sciences \\ Loughborough University \\ R.H.J.Grimshaw@lboro.ac.uk
}

Abstract When a fluid flow interacts with a topographic feature, and the fluid can support wave propagation, then there is the potential for waves to be generated upstream and/or downstream. In many cases when the topographic feature has a small amplitude the situation can be successfully described using a linearised theory, and any nonlinear effects are determined as a small perturbation on the linear theory. However, when the flow is critical, that is, the system supports a long wave whose group velocity is zero in the reference frame of the topographic feature, then typically the linear theory fails and it is necessary to develop an intrinsically nonlinear theory. It is now known that in many cases such a transcritical, weakly nonlinear and weakly dispersive theory leads to a forced Korteweg- de Vries (fKdV) equation. In canonical form, this is given by

$$
-u_{t}-\Delta u_{x}+6 u u_{x}+u_{x x x}+f_{x}=0,
$$

where $u(x, t)$ is the amplitude of the critical mode, $t$ is the time coordinate and $x$ is the spatial coordinate, $\Delta$ is the phase speed of the critical mode, and $f(x)$ is a representation of the topographic feature.

In this article we shall sketch the contexts where the fKdV equation is applicable, and describe some of the most relevant solutions. There are two main classes of solutions. In the first, the initial condition for the $\mathrm{fKdV}$ equation is $u(x, 0)=0$ so that the waves are generated directly by the flow interaction with the topography. In this case the solutions are characterised by the generation of upstream solitary waves and an oscillatory downstream wavetrain, with the detailed structure being determined by $\Delta$ and the polarity of the topographic forcing term $f(x)$. In the second class a solitary wave is incident on the topography, and depending on the system parameters may be repelled with a significant amplitude change, trapped with a change in amplitude, or allowed to pass by the topography with only a small change in amplitude. 


\section{INTRODUCTION}

Scattering of waves by obstacles, that is, inhomogeneous features in an otherwise homogeneous waveguide, is a much-studied problem with a long history. For the most part, linear theories are appropriate and have been very successful, in spite of the often very complicated wave patterns that can be formed. In this context nonlinear effects are then calculated as a second-order perturbation, and although such calculations, often numerical, can reveal new physical effects such as wave-induced mean flows or long-time wave-packet modulation, it remains the case that the first-order understanding arises from linear theory. However, there exist special but important circumstances when linear theory fails and nonlinearity enters at the first order. Two situations can be identified when this occurs. The first is when the incident wave field is itself nonlinear; an extreme instance would be when the incident wave is a shock wave. The second case is the subject of this article. Consider the situation when the waveguide can support a wave mode with zero group velocity in the frame of reference of the obstacle. In this situation, wave energy cannot readily escape from the obstacle and the wave amplitude in a linear theory grows indefinitely with time. An intrinsically nonlinear theory is then needed, even when the incident wave has ony a small amplitude. In this article we will sketch some typical circumstances where this arises, and the emergence of the forced Korteweg-de Vries equation, or related equations, as a paradigm for nonlinear scattering of long waves.

Consider then a fluid flow interacting with a topographic feature, in circumstances where the fluid supports wave propagation. A typical example is water in a horizontal channel; other examples are provided by the stable density stratification of shallow coastal seas, or the boundary layer in the lower atmosphere. For simplicity, we shall discuss here mainly the important case when the waveguide has only one active spatial dimension, and the waves are long waves, that is their wavelengths are much greater than a typical transverse length scale associated with the waveguide. There is then the potential for waves to be generated upstream and/or downstream. Linear theory can successfully be used here when the topographic feature has a small amplitude and all waves in the system have finite, non-zero group velocity in the frame of reference of the obstacle. However, when the flow is critical, that is, the system supports a long wave whose group velocity is close to zero in the reference frame of the obstacle, then it is necessary to develop an intrinsically nonlinear theory. It is now known that in many cases such a transcritical, weakly nonlinear and weakly dispersive theory leads to a 
forced Korteweg-de Vries equation. In canonical non-dimensional form, this is given by

$$
-u_{t}-\Delta u_{x}+6 u u_{x}+u_{x x x}+f_{x}(x)=0,
$$

where $u(x, t)$ is the amplitude of the critical mode, $t$ is the time coordinate and $x$ is the spatial coordinate, $\Delta$ is the long-wave speed of the critical mode, and $f(x)$ is a representation of the topographic obstacle.

Explicit asymptotic derivations of the fKdV equation (1.1) have been carried out for water waves in a channel $([1,4,13,18])$, internal waves in a shallow fluid $([7,14,2,3])$, inertial waves in a narrow tube $[6]$ and topograhic waves in an oceanic coastal waveguide $([5,15]$, along with analogous derivations in several other physical contexts. Here we sketch a schematic derivation which indicates how a more formal derivation might proceed. Suppose that in linear long wave theory there is a wave mode whose group velocity $\Delta$ is close to zero in the reference frame of the obstacle. Then using a standard linear long wave theory, the amplitude $u(x, t)$ of this mode can be shown to satisfy the forced first-order wave equation

$$
u_{t}+\Delta u_{x}=f_{x}(x),
$$

where $\left.f_{(} x\right)$ is a typically a projection of the actual obstacle profile onto the wave mode in question. The solution of equation (1.2) for the zero initial condition $u(x, 0)=0$ is given by

$$
u=\frac{f(x)-f(x-\Delta t)}{\Delta}, \text { if } \Delta \neq 0, \text { or } u=t f_{x}(x) \text { if } \Delta=0 .
$$

Clearly this solution fails as $\Delta \rightarrow 0$. In this case it is necessary to add a nonlinear term, which is generically quadratic and of the form given in (1.1); further it is now well-understood that when adding a weakly nonlinear correction it is necessary to add a balancing weakly linear dispersive term, which typically has the form given in (1.1). Formally this is achieved by using a multi-scale asymptotic expansion where $\partial / \partial x \sim \epsilon$, $\partial / \partial t \sim \epsilon^{3}, u \sim \epsilon^{2}, \Delta \sim \epsilon^{2}$ and $f \sim \epsilon^{4}$. There are plenty of illustrations in the afore-mentioned references to explicit derivations. One of the most important features of this fKdV model emerges already from this scaling, in that the response to a small forcing of $O(f)$ is $O\left(f^{1 / 2}\right)$.

Before proceeding we need to describe the steady-state solutions of unforced KdV equation (i.e (1.1) with $f \equiv 0$ ). First there is the wellknown solitary wave,

$$
\text { where } \quad \begin{aligned}
u & =a \operatorname{sech}^{2} \beta(x-V t), \\
\Delta-V & =2 a=4 \beta^{2} .
\end{aligned}
$$


Next, there are the periodic solutions, namely the so-called "cnoidal" waves, given by

$$
\begin{aligned}
u & =a\left\{b(m)+\operatorname{cn}^{2} \beta(x-V t)\right\}+d, \\
\text { where } \quad b & =\frac{1-m}{m}-\frac{E(m)}{m K(m)}, \\
\Delta-V & =6 d+2 a\left\{\frac{2-m}{m}-\frac{3 E(m)}{m K(m)}\right\}, \\
\text { and } \quad a & =2 m \beta^{2} .
\end{aligned}
$$

Here $\operatorname{cn}(x)$ is the Jacobian elliptic function of modulus $m$, while $K(m)$ and $E(m)$ are the complete elliptic integrals of the first and second kind respectively. The mean value of $A$ over one period is $d$, while the spatial period is $2 K(m) / \beta$. As $m \rightarrow 1, \mathrm{cn}^{2}(x) \rightarrow \operatorname{sech}^{2}(x), b(m) \rightarrow 0$, and then (1.5) becomes solitary wave (1.4, relative to the level $d$. As $m \rightarrow 0$, $b+\operatorname{cn}^{2}(x) \rightarrow \cos 2(x), a \rightarrow 0$ and $V \rightarrow \Delta-6 d+4 \beta^{2}$; this is just a sinusoidal wave train relative to the level $d$.

In this article we shall describe some of the most relevant solutions of equation (1.1). There are two main classes of solutions. In the first, discussed in Section 2, the initial condition for $(1.1)$ is $u(x, 0)=0$ so that the waves are generated directly by the flow interaction with the topography. In this case the solutions are characterised by the generation of upstream solitary waves and an oscillatory downstream wave train, with the detailed structure being determined by $\Delta$ and the polarity of the topographic forcing term $f(x)$. In the second class, discussed in Section 3, a solitary wave is incident on the topography, and depending on the system -parameters may be repelled with a significant amplitude change, trapped with a change in amplitude, or allowed to pass by the topography with only a small change in amplitude.

\section{GENERATION OF UPSTREAM AND DOWNSTREAM WAVES}

Here we consider the case when the waves are generated directly by the topography, when the appropriate initial condition for equation (1.1) is that $u(x, 0)=0$. A set of typical solutions of are shown in Figure 1 for the case when the forcing term is positive and isolated. That is $f(x)$ is positive, and non-zero only in a vicinity of $x=0$, with a maximum value of $f_{M}$. For exact criticality, when $\Delta=0$ (Figure 1a) the solution is characterised by upstream and downstream wavetrains connected by a locally steady solution over the obstacle. When the oncoming flow is subcritical, so that $\Delta<0$ (Figure 1b), the upstream wavetrain weakens, and for 
sufficiently large $|\Delta|$ detaches from the obstacle, while the downstream wavetrain intensifies and for sufficiently large $|\Delta|$ forms a stationary lee wave field. When the oncoming flow is supercritical, so that $\Delta>0$ (Figure 1c) the upstream wavetrain develops into well-separated solitary waves, while the downstream wavetrain weakens and moves further downstream. For more details, see $[7,16]$.

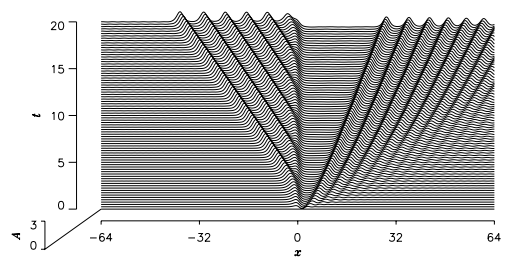

(a) $\Delta=0$

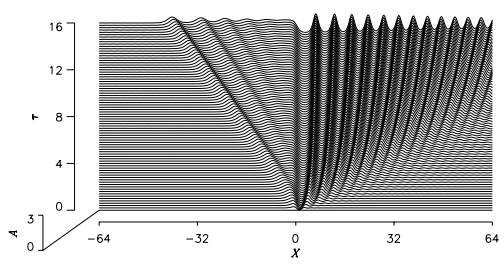

(b) $\Delta<0$

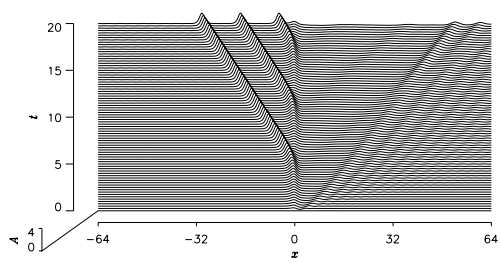

(c) $\Delta>0$

Figure 1: Typical solutions of the forced KdV equation (1.1) for (a) $\Delta=0$, (b) $\Delta<0$ and (c) $\Delta>0$. 
The origin of the upstream and downstream wavetrains can be found in the structure of the locally steady solution over the obstacle. In the transcritical régime this is characterised by a transition from a constant state $U_{-}$upstream of the obstacle to a constant state $U_{+}$downstream of the obstacle, where $U_{-}>0$ and $U_{+}<0$. It is readily shown that $\Delta=3\left(U_{+}+U_{-}\right)$independently of the details of the forcing term $f(x)$. Explicit determination of $U_{+}$and $U_{-}$requires some knowledge of the forcing term $f(x)$. However, in the "hydraulic" limit when the linear dispersive term in (1.1) can be neglected, it is readily shown that

$$
6 U_{ \pm}=\Delta \mp\left(12 f_{M}\right)^{\frac{1}{2}} .
$$

This expression also serves to define the transcritical régime, which is

$$
|\Delta|<\left(12 f_{M}\right)^{\frac{1}{2}}
$$

Thus upstream of the obstacle there is a transition from the zero state to $U_{-}$, while downstream the transition is from $U_{+}$to 0 ; each transition is effectively generated at $x=0$.

Both transitions are resolved by "undular bore" solutions. That is, we use the Whitham modulation theory $([17,12])$ in which the periodic wavetrain (1.5) is allowed to have a slowly-varying amplitude $a$, modulus $m$ and mean depth $d$. For the undular bore solution, these are functions of the similarity variable $x / t$. The downstream "undular bore" is then given by

$$
\begin{array}{r}
\Delta-\frac{x}{t}=2 U_{+}\left\{2-m+\frac{2 m(1-m)(K(m)}{E(m)-(1-m) K(m)}\right\} \\
\text { for } \quad \max \left\{0, \Delta-2 U_{+}\right\} \quad<\frac{x}{t}<\Delta-12 U_{+}, \\
a=-2 U_{+} m, \quad \text { and } \quad d=U_{+}\left\{2-m-\frac{2 E(m)}{K(m)}\right\} .
\end{array}
$$

Ahead of the wavetrain where $x / t \approx \Delta-12 U_{+}, m \rightarrow 0$ and the waves are approximately sinusoidal. Behind the wavetrain where $x / t \approx \Delta-$ $2 U_{+}, m \rightarrow 1$ and the waves are approximately solitary waves of amplitude $-2 U_{+}$relative to the mean level of $U_{+}$. Further, it can be shown that on any individual crest in the wavetrain, $m \rightarrow 1$ as $t \rightarrow \infty$. In this sense, the undular bore evolves into a train of solitary waves. This downstream wavetrain is constrained to lie in $x>0$, and hence is only fully realised if $\Delta>2 U_{+}$. Combining this criterion with (2.1) and (2.2) then leads to the régime,

$$
\left.\left.-\frac{1}{2}\left(12 f_{M}\right)^{\frac{1}{2}}\right)<\Delta<\left(12 f_{M}\right)^{\frac{1}{2}}\right)
$$


On the other hand, when $\Delta<2 U_{+}$, we obtain the régime,

$$
\left.\left.-\left(12 f_{M}\right)^{\frac{1}{2}}\right)<\Delta<-\frac{1}{2}\left(12 f_{M}\right)^{\frac{1}{2}}\right) .
$$

Now the downstream undular bore is attached to the obstacle, with a modulus $m_{s}(<1)$ at the obstacle, where $m_{s}$ can be founding by putting $x=0$ in (2.3). Indeed, a stationary lee wavetrain develops just behind the obstacle (for further details, see [16]).

The upstream "undular bore" is given by

$$
\begin{array}{r}
\Delta-\frac{x}{t}=2 U_{-}\left\{1+m-\frac{2 m(1-m)(K(m)}{E(m)-(1-m) K(m)}\right\} \\
\text { for } \quad \max \left\{0, \Delta-4 U_{-}\right\} \quad<\frac{x}{t}<\Delta+6 U_{-}, \\
a=2 U_{-} m, \quad \text { and } \quad d=U_{-}\left\{m-1+\frac{2 E(m)}{K(m)}\right\} .
\end{array}
$$

Ahead of the wavetrain where $x / t \approx \Delta-4 U_{-}, m \rightarrow 1$ and the waves are approximately solitary waves of amplitude $2 U_{-}$where the leading wave has an amplitude of $2 U_{-}$. Behind the wavetrain where $x / t \approx$ $\Delta-6 U_{-}, m \rightarrow 0$ and the waves are approximately sinusoidal. Further, as before, it can be shown that on any individual crest in the wavetrain, $m \rightarrow 1$ as $t \rightarrow \infty$, so that in this sense, he undular bore evolves into a train of solitary waves. This upstream wavetrain is constrained to lie in $x<0$, and hence is only fully realised if $\Delta<-6 U_{-}$. Combining this criterion with (2.1) and (2.2) then leads to the régime (2.5). Thus a fully detached upstream "undular bore" coincides with the case when the downstream "undular bore" is attached. On the other hand, when $\Delta>$ $-6 U_{-}$, we obtain the régime (2.4), and so a fully detached downstream "undular bore" coincides with the case when the upstream "undular bore" is attached. It has a modulus $m_{0}$ at the obstacle, where $m_{0}$ is determined by putting $x=0$ in (2.6), see [16] for further details.

For the case when the obstacle provides a negative, but still isolated, forcing term (i.e. $f(x)$ is negative, and non-zero only in the vicinity of $x=0$ ), the upstream and downstream solutions are qualitatively similar to those described above for positive forcing. However, the solution in the vicinity of the obstacle remains transient, and this causes a modulation of the "undular bore" solutions.

\section{INTERACTION OF A SOLITARY WAVE WITH AN OBSTACLE}

Here we consider the situation when a solitary wave is incident on the topographic obstacle. As in the previous section, we shall suppose 
that the obstacle is isolated, but may have either polarity. The initial condition is a free solitary wave located far from the obstacle. That is, $u(x, 0)$ is given by (1.4) with $t=0$ and $x$ replaced by $x-x_{0}$ where $x_{0}$ is such that $f\left(x_{0}\right) \approx 0$. As the solitary wave propagates towards the obstacle its speed $V$ will adjust, and we would expect either trapping if $V \rightarrow 0$ as $t \rightarrow \infty$, or repulsion if $V$ passes through zero and changes sign. Both these cases clearly require that $\Delta>0$, i.e. the flow is supercritical. Otherwise, if $V$ retains the same sign throughout the interaction, then we have passage. Clearly, this will always be the case if $\Delta<0$, i.e the flow is subcritical, since then $V$ remains negative throughout the interaction. The passage regime may also ocur if $\Delta>0$ provided that either the solitary wave amplitude $a$ remains large so that $V$ remains negative, or $a$ remains small, so that $V$ remains positive.

These notions can be quantified by assuming that the obstacle provides only a small, and slowly-varying, effect on the solitary wave. Thus, we assume that locally the solitary wave retains its shape, but its amplitude and speed vary slowly. The derivation of the equations governing the amplitude is a multi-scale asymptotic procedure. As this is wellknown (see, for instance, [8]), and has been described for the present circumstances in [9], we shall omit all details here. The outcome is that the solitary wave is described by,

$$
\begin{gathered}
u=\operatorname{asech}^{2}(\beta(t) \Phi), \\
\text { where } \quad \Phi(t)=x-\Psi(t), \quad \Psi(t)=\int_{0}^{t} V\left(t^{\prime}\right) d t^{\prime}, \\
\text { and } \quad \Delta-V=2 a=4 \beta^{2} .
\end{gathered}
$$

The evolution of the amplitude is determined essentially by an "energy" equation, which here takes the form,

$$
\frac{d a}{d t}=\beta \int_{-\infty}^{\infty} \operatorname{sech}^{2}(\beta \Phi) \frac{\partial f}{\partial \Phi}(\Phi+\Psi) \Phi .
$$

A second equation is obtained from the leading order relationship between the speed and the amplitude in (3.1), which is

$$
V=\frac{d \Psi}{d t}=\Delta-2 a \text {. }
$$

Together, (3.2) and (3.3) form a coupled system of ordinary differential equations for the amplitude $a$ and position $\Psi$ of the solitary wave. This system has been discussed in detail by [9] who also include the effects of higher-order corrections to the "speed" equation (3.3). The effects of allowing $\Delta$ to vary slowly in time have been discussed in [10], while the effects of including some dissipation were discused in [11]. 
In order to obtain some simple formulas, we shall here make the further approximation that the solitary wave is much narrower than the obstacle. This is the so-called "broad" forcing case of [9], which they showed to be representative. In this limit, equation (3.2) becomes

$$
\frac{d a}{d t}=2 \frac{\partial f}{\partial \Phi}(\Phi)
$$

The system consisting of (3.4) and (3.3) is Hamiltonian with the Hamiltonian

$$
H=\Delta a-a^{2}-2 f(\Psi) .
$$

$H$ is an invariant, and the orbits are given by $H=$ constant. We will consider here symmetric and isolated functions $f(\Psi)$ with a single stationary value at $\Psi=0$ of $f_{M}$ which is a maximum (minimum) for $f_{M}>0(<0)$. Then the system $(3.4),(3.3)$ has a single critical point at $\Psi=0$ and $a=a_{c}=\Delta / 2$. Since $a$ is constrained to remain positive, this critical point does not exist if $\Delta<0$. In this latter case all the orbits pass from $\Psi=\infty$ to $\Psi=-\infty$ as $t$ increases, which corresponds to a passage regime. Otherwise, when $\Delta>0$ the critical point is a centre when $f_{M}<0$ and so the obstacle is positive (that is, it has the same polarity as the solitary wave), while the critical point is a saddle point when $f_{M}>0$ and so the obstacle is negative (that is, it has the opposite polarity to the solitary wave).

In the case of a centre, there is a family of periodic orbits surrounding the critical point, corresponding to the trapping regime. The critical point represents a stationary solitary wave trapped at the obstacle location. This family of periodic orbits is contained within a pair of "homoclinic" orbits, which pass to infinity at the value $a=a_{\infty}=a_{c}$ (that is, at the same amplitude as that at the critical point), and reach maximum (minimum) amplitudes of $a_{c} \pm\left(-2 f_{M}\right)^{1 / 2}$ at $\Psi=0$ (that is, at the location of the obstacle maximum). Note that if $\Delta^{2}<-8 f_{M}$, then the minimum amplitude is less than zero, and so cannot be realised (but see [9]) for the resolution of this dilemma). All orbits outside these "homoclinic" orbits correspond to the passage regime, with those orbits whose amplitude at infinity is greater (less) than $a_{c}$ passing from right to left (left to right).

For the case of a saddle point, there are "homoclinic" orbits emanating from the saddle point, these being two unstable manifolds and two stable manifolds. These pass to infinity with an amplitude $a=a_{\infty}=$ $a_{c} \pm\left(2 f_{M}\right)^{1 / 2}$. Note that if $\Delta^{2}<8 f_{M}$, then the smaller of these amplitudes is less than zero, and so cannot be realised (again, see [9]) for the resolution of this dilemma). All orbits which at infinity have an amplitude lying between these two values correspond to the repulsion regime. 
Those orbits starting from the left (right) show an increase (decrease) in amplitude. All orbits whose amplitude at infinity lies outside this range correspond to a passage regime, and those orbits with the smaller (larger) amplitude pass from left to right (right to left) in the sense of increasing $\Psi$.

These typical scenarios have been confirmed by numerical simulations of the fKdv equation (1.1) reported in [9]. Here we show two typical results in Figure 2, these being (a) for a trapping regime, and (b) for a repulsion regime. Other similar results are shown in [9].

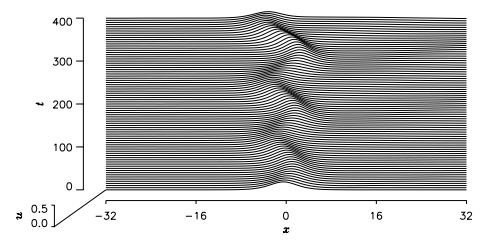

(a) $f_{M}<0$

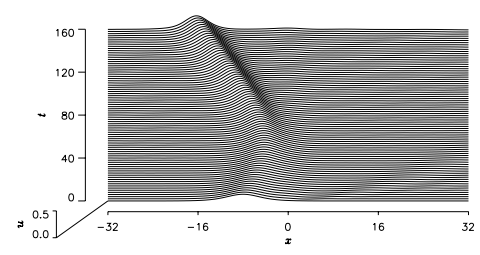

(b) $f_{M}>0$

Figure 2: Typical solutions of the forced KdV equation (1.1) for the case of a solitary wave incident on an obstacle; (a) a trapping regime when $f_{M}<0$, a repulsion regime when $f_{M}>0$.

\section{References}

[1] Akylas T.R. (1984) On the excitation of long nonlinear water waves by moving pressure distribution. J.Fluid Mech., 141, 455-466. 
[2] Clarke, S.R. and Grimshaw, R.H.J. (1994). Resonantly generated internal wave in a contraction. J. Fluid Mech., 274, 139-161.

[3] Clarke, S.R. and Grimshaw, R.H.J. (2000). Weakly-nonlinear internal wave fronts trapped in contractions. J. Fluid Mech., 415, 323-345.

[4] Cole S.L. (1985). Transient waves produced by flow past a bump. Wave Motion , 7, 579-587.

[5] Grimshaw R. (1987). Resonant forcing of barotropic coastally trapped waves. J.Phys. Oceanogr., 17, 53-65.

[6] Grimshaw R.(1990). Resonant flow of a rotating fluid past an obstacle: the general case. Stud. Appl.Maths., 83, 249-269.

[7] Grimshaw R. and Smyth N. (1986). Resonant flow of a stratified fluid over topography. J.Fluid Mech., 169, 429-464.

[8] Grimshaw, R. and Mitsudera, H. (1993). Slowly-varying solitary wave solutions of the perturbed Korteweg-de Vries equation revisited. Stud. Appl. Math., 90, 75-86.

[9] Grimshaw, R., Pelinovsky, E. and Tian, X. (1994). Interaction of a solitary wave with an external force. Physica D, 77, 405-433.

[10] Grimshaw, R., Pelinovsky, E. and Sakov, P. (1996). Interaction of a solitary wave with an external force moving with variable speed. Stud. Appl. Math., 97, 235-276.

[11] Grimshaw, R., Pelinovsky, E. and Bezen, A. (1997). Hysteresis phenomena in the interaction of a damped solitary wave with an external force. Wave Motion, 26, 253-274.

[12] Gurevich, A.V. and Pitaevskii, L.P. (1974). Nonstationary structure of a collionsless shock wave. Sov. Phys. JETP, 38, 291-297.

[13] Lee S.J., Yates G.T., and Wu T.Y. (1989). Experiments and analyses of upstream-advancing solitary waves generated by moving disturbances. J.Fluid Mech., 199, 569-593.

[14] Melville W.K. \& Helfrich K.R.(1987). Transcritical two-layer flow over topography. J.Fluid Mech., 178, 31-52.

[15] Mitsudera H. \& Grimshaw R. (1990). Resonant forcing of coastally trapped waves in a continuously stratified ocean. Pageoph., 133, 635644.

[16] Smyth N. (1987). Modulation theory solution for resonant flow over topography. Proc. Roy. Soc. London, A409, 79-97.

[17] Whitham, G.B. (1974). Linear and Nonlinear Waves, Wiley, New York, pp 636.

[18] Wu T.Y. (1987). Generation of upstream advancing solitons by moving disturbances. J.Fluid Mech., 184, 75-99. 